\title{
Establishing an efficient regulatory mechanism-Prerequisite for successful energy activities regulation
}

\author{
Eraldo Banovac ${ }^{\mathrm{a}, *}$, Mevludin Glavic $^{\mathrm{b}}$, Sejid Tešnjak ${ }^{\mathrm{c}}$ \\ a The Steering Committee of the Croatian Energy Regulatory Agency, Koturaška 51, 10000 Zagreb, Croatia \\ ${ }^{\mathrm{b}}$ Electrical Engineering and Computer Science Department, University of Liege, B-4020 Liege, Belgium \\ ${ }^{\mathrm{c}}$ Department of Power Systems, Faculty of Electrical Engineering and Computing, University of Zagreb, Unska 3, 10000 Zagreb, Croatia
}

\section{A R T I C L E I N F O}

\section{Article history:}

Received 11 September 2007

Available online 24 January 2009

Keywords:

Model of the regulatory system

Regulation

Regulatory mechanism

System entropy

\begin{abstract}
A B S T R A C T
An analytic approach to determine appropriate regulatory strategies for the energy sector is proposed in this paper. A basic model of the regulatory system in the energy sector is defined, and the regulatory mechanism functions in this context are outlined. The basic problem of unknown factors (i.e., system entropy) is highlighted. An original algorithm developed to analyze regulatory background context and regulatory mechanism functions is discussed. A useful method for defining existing level of energy activities regulation is also presented using the Croatian regulatory framework as an example.
\end{abstract}

(c) 2008 Elsevier Ltd. All rights reserved.

\section{Introduction}

Appropriate regulation of energy activities has become increasingly important over the past decade. More than a hundred countries have regulatory bodies that oversee energy activities. There exists no universally accepted approach. Indeed, it is preferable for each country to develop its own model of regulation that takes into account actual economic conditions and the nature of the national energy sector. In general, models should also reflect the increasing globalization and liberalization of energy markets across the world. The models should also reflect certain basic principles of energy regulation, and they should provide a transparent framework within which regulatory authorities can operate.

Regulation can have both positive and negative impacts. Ideally, regulation should protect consumers from monopolies and ensure that they have sufficient information to make informed decisions. In this manner, appropriate regulation can contribute to a more equitable and productive society. However, regulation can also have certain disadvantages. For example, it may impose burdensome informational requirements, cause unforeseen distortions in the energy market, or involve lengthy and costly regulatory procedures. In addition, regulation frameworks may provide insufficient guidance as to what constitutes anti-competitive practices among market-leading energy providers, and this can lead to market uncertainty. From the

\footnotetext{
* Corresponding author. Tel.: +38516311412; fax: +38516311344.

E-mail addresses: eraldo.banovac@zg.t-com.hr, eraldo.banovac@zg.htnet.hr (E. Banovac).
}

consumer's perspective, regulation can suffer from negative press and poor public acceptance.

Despite the potential problems of regulation, an unregulated energy market is also clearly problematic. Specifically, a lack of regulation may lead to companies' imposing costs on their customers that are not consistent with the expenses that the companies incur in purchasing the energy and providing it to the client. Similarly, an unregulated energy market often leads to unacceptable production of pollutants. Finally, without regulation, companies are not incentivized to provide consumers with the information necessary to make reasonable choices.

Breaking monopolies and privatizing energy markets can significantly impact national economies. Accordingly, it is in the national interest of every country to:

- Develop longer-term regulation strategies that look at least 15 years into the future.

- Support analytic approaches to regulation.

- Introduce transparent rules for energy operations that reflect the importance of energy to the national economy.

Moreover, the scope of action for regulatory authorities should be defined clearly. Generally, an efficient and incentive-based regulatory environment can be guaranteed if the authorities:

- act to counter monopoly-like activities;

- regulate the prices of energy and ancillary services;

- ensure equal access to the grid;

- define fees for access to and usage of the grid; 


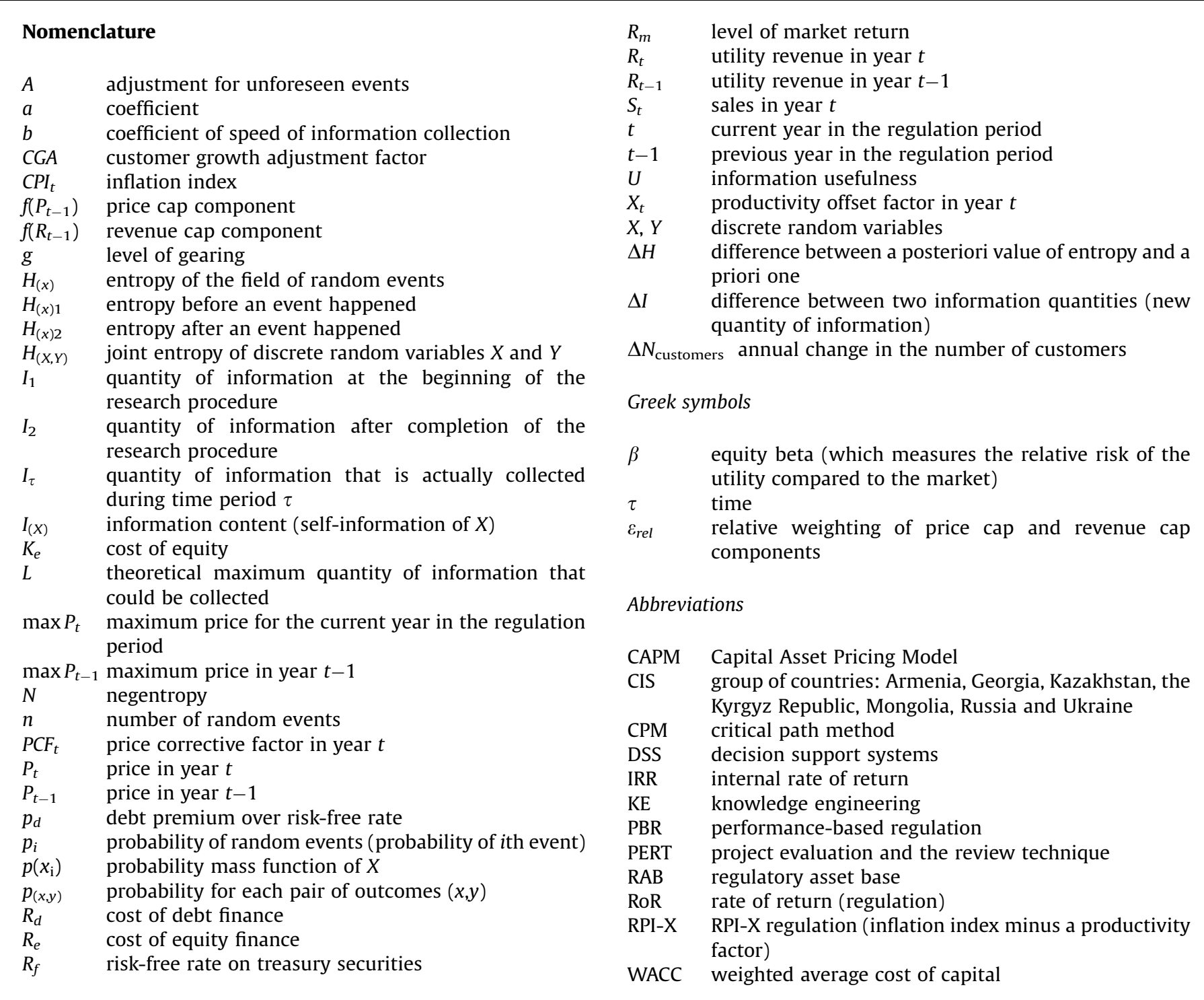

- issue licenses and take action in cases of non-compliance with the terms of a license, especially when these impact safety;

- ensure adequate separation (unbundling) between different energy activities performed by a given vertically integrated energy provider/corporation;

- perform conflict resolution between energy market participants;

- protect customers;

- ensure that regulatory activities comply with relevant legislation;

- incentivize investment.

The principles described above may seem obvious, but substantial effort is required to implement such ideas. To sustain this reason, a situation regarding main problems that regulation has had in Croatia should be shortly explained. The institutional strengthening of entitled regulatory body had not developed satisfactory, especially from 2002 to 2006 because the regulatory body had insufficient expertise and the Energy institute prepared analyses for regulatory decisions. Establishment of the regulatory authority took far too long. The regulatory organization was chronically short-staffed and therefore unable to meet all of its obligations. The own premises of regulatory body have not been ensured for years. The fee for financing the regulatory doing was decreased in June 2005 following a government decision. Due to described situation the question of how to re-establish an efficient regulatory system has become very important. The authors of this paper would advocate the use of a research-based process to explore this question. The authors propose a methodology that includes system modeling, outlining the basic regulatory functions, and developing an efficient organization.

In this paper, a basic model of the regulatory system and an algorithm to efficiently describe required regulatory mechanism functions are introduced. The algorithm is applicable at the level of a national energy sector, and it can therefore be tailored to meet specific regulatory objectives. It is necessary to determine which regulatory mechanism functions should be investigated for each specific national energy sector. The number of functions described can be adjusted as necessary, but it is generally between 4 and 6 . The algorithm presented in this paper is based on the regulatory mechanism that is a part of the nominated basic model of the regulatory system. The algorithm is consistent with information theory research [1-3]. Its general purpose is to provide new information as a means of decreasing the entropy of an existing regulatory system.

The paper is organized as follows. In section 2, the regulatory mechanism is overviewed. Section 3 introduces and defines 
entropy in this context. A specific algorithm for analyzing the regulatory background context and the regulatory mechanism functions is given in Section 4. As a case study, the algorithm with four regulatory mechanism functions is applied to the aforementioned Croatian scenario. Furthermore, a project of new organization of the regulatory body is nominated in this section. Section 5 discusses the intensity of implemented energy activities regulation, while Section 6 offers some conclusions.

\section{Regulatory mechanism}

The regulation refers to the set of laws, sub-laws and methods, the general aim of which is to improve the decisions made by companies and individuals in the energy sector. An additional aim may be to lower the cost of regulated activities while maintaining the quality of delivered energy and protecting the environment. To achieve these goals requires the use of modern management techniques, together with sufficient financial backing and competent staff.

An energy system should be defined before making any analysis regarding regulatory mechanism's issues. It can be defined as an "open system", a term that is commonly used in both thermodynamics and systems theory. This open system can be described in terms of its main characteristics-environment, input, throughput, output and feedback for self-control (Fig. 1). Certain external components can impact system performance. Among the most influential are consumers, regulation, energy market, etc. The term "input" refers to source of information and resources that come from system environment and allow the system to function. The term "throughput" indicates the process of transformation/conversion of resources within the system. The "output" is the result of the system's operation; in the case of energy, it is often a product such as electricity that is exported back to the environment.

In this paper, the basic model of the regulatory system in the energy sector including a specific regulatory mechanism is set. The term "regulatory mechanism" refers to the means by which the following functions are achieved:

(1) Licensing: the process of issuing licenses to energy undertakings ${ }^{1}$ for carrying out certain activities.

(2) Monitoring 2 : control and inspection of the aforementioned activities. It includes supervising technical and financial performance, as well as ensuring compliance with requirements for staff professional qualifications. It also includes collecting economic data such as prices, costs and revenues, together with general market assessments.

(3) Setting and implementing tariffs: this is linked to price regulation.

(4) Customer protection: this is related to efficient dispute resolution and maintenance of quality of supply.

To achieve the aforementioned functions requires carefully prescribed methods. These methods can be selected by assessing the following:

- which elements are part of the existing regulatory framework;

- national energy market characteristics;

- definitions of regulatory success;

- core competencies of the existing regulatory body;

\footnotetext{
${ }^{1}$ The term "energy undertaking" refers to a legal or physical person carrying out one or more energy-related activities.

${ }^{2}$ As described in [4].
}

- types of licensing;

- chosen regulatory monitoring framework;

- tariff mechanisms;

- policy regarding customer protection.

Establishing regulatory mechanism functions should be based on an analytic approach, using an investigative approach to explore the broader context of the regulatory acting. The latter is important because there is often insufficient contextual knowledge within the regulatory authorities themselves.

The system control function that influences regulatory mechanism is shown in Fig. 2. The purpose of the system is to keep the regulatory framework operational. The control function is selfregulated and is connected with system input and output via both feedforward and feedback loops. In practice, national legislation compels regulatory authorities to perform these functions. Moreover, there is an audit function in the system environment that influences the control function (regulatory body). This function is performed in practice by policymakers and legislative bodies. In the Croatian case study, energy legislation demarcates the connections between the Croatian Parliament, the Government of the Republic of Croatia and the regulatory body. The Charter of the regulatory body can only be modified with prior Governmental approval. The commissioners are appointed and discharged by the Croatian Parliament upon the Government's request. The regulatory body may not acquire, encumber or dispose of any property exceeding one half of the regulatory budget without the consent of the Government. The Government exercises founding rights in relation to the regulatory body. Furthermore, lawmakers are obligated to request the opinion of the regulatory body on draft proposals of energy-related legislation. The Government gives a prior opinion on an annual budget of the regulatory body. The regulatory body is obliged to submit an annual report on its activities, in addition to reports on specific issues at more frequent intervals. These reports are presented to the Croatian Parliament or to the Government if so requested.

\subsection{Input and output of the regulatory system}

The system input includes:

- Legislation, namely, national energy laws. In the case of European nations, relevant legislation may also include EU Directives that prescribe harmonized rules for internal electricity and gas markets.

- Sub-laws, namely, grid code, market code, general conditions of energy supply, tariff systems, etc.

- Rules, methods and regulation techniques.

- Standards of operation.

- Significant information and energy required for system operation.

The system output contains information essential for system operation and maintenance.

From a theoretical standpoint, the regulatory system can be considered a closed dynamic system. The output can influence the input via feedback, which drives the system toward appropriate results. Feedback operations include:

- Measuring the output.

- Comparing actual results with the expected ones.

- Conducting analysis.

- Controlling the system (action). 
SY S TEM ENVIRONMENT

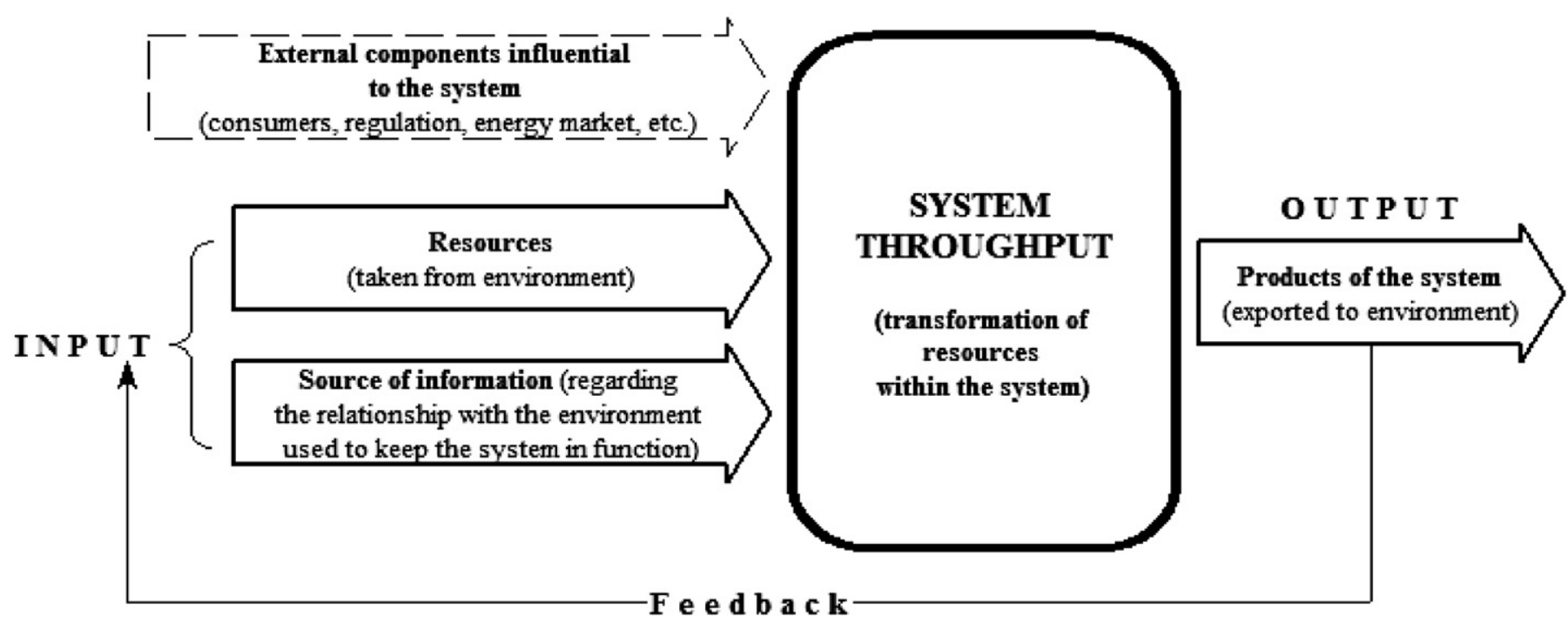

Fig. 1. Structure of an energy system.

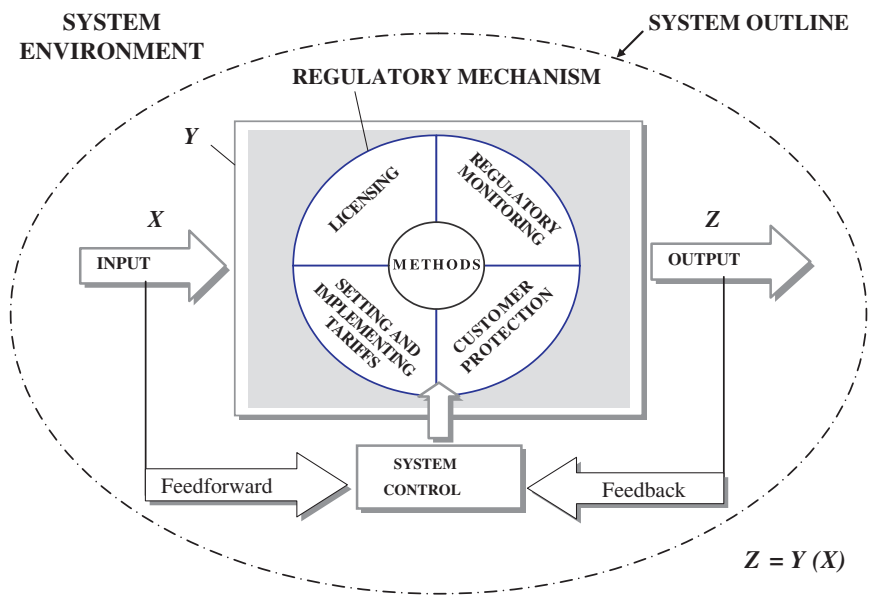

Fig. 2. Basic model of the regulatory system.

\section{Entropy of the regulatory system}

An argument could be made for the elimination of energy as a system input, since the considered theoretical framework does not address production. Legislation and associated rules/techniques clearly can and should be defined in advance. Accordingly, information is the only unknown factor among the system inputs. The unknown component of the input-output relationship depends on system entropy, since the success of each system operation increases total regulatory knowledge. That knowledge is the sum of all the information that exists in the energy regulatory field. This leads to the conclusion that information can be defined as anything that increases total knowledge (from system input) or knowledge about a certain event (from system output). Information is the measure used to classify system state.

It is important to distinguish between information and informativity. Generally, a significant amount of information is collected, processed and analyzed in these types of research problems as a means of increasing informativity. However, to achieve good informativity, it is necessary to choose the correct option among multiple possibilities that may initially appear indistinguishable in terms of value. Accordingly, it is critical to remember that information as defined in information theory constitutes only a small part of informativity.

In order to decrease the level of uncertainty (and therefore entropy) in a regulatory system, it is important to either reorganize the system or bring in new information. Entropy can be determined as follows:

(1) Defining all the cases that the system can fulfill.

(2) Defining initial probabilities.

(3) Investigating connections related to each case occurrence.

The system entropy is a measure of the uncertainty associated with a random variable. According to probability theory, the variable is a quantity whose values are random and to which a probability distribution is assigned. The random variable is a measurable function that maps from a sample space to the measurable space of possible variable values. The system entropy is the same as the information quantity necessary to cancel the uncertainty of a given event result. Accordingly, system entropy essentially quantifies the information associated with a certain piece of data. According to [1,2], the system (Shannon or information) entropy is expressed as

$H_{(x)}=-\sum_{i=1}^{n} p_{i} \log _{2}\left(p_{i}\right) \quad$ (bit)

where $H_{(x)}$ is entropy of the field of random events, $n$ is the number of random events, and $p_{i}$ is the probability of random events (probability of $i$ th event). The probability of an event occurring is defined as the number of cases that result in the event, over the number of total outcomes possible in an equiprobable sample space.

A minus operator in (1) ensures that the calculated value is positive. Eq. (1) implies that information quantity exactly sufficient to cancel an event's uncertainty ${ }^{3}$ is equal to the entropy of the field/system that contains all possible events. A simple

\footnotetext{
${ }^{3}$ This means that the result of an event is completely known.
} 
example provides a helpful illustration. There are six equiprobable random events in the case of throwing the dice, i.e., $n=6$ and $p_{i}=\frac{1}{6}$. Knowing that $\mathrm{ld}=\log _{2}$, the entropy for this case is calculated as

$H_{(x)}=-\sum_{i=1}^{6} \frac{1}{6} \operatorname{ld} \frac{1}{6}=-\operatorname{ld} \frac{1}{6}=-(\operatorname{ld} 1-\operatorname{ld} 6)=2.585 \quad$ (bit)

In general, given a random variable $X$, the entropy $H_{(X)}$ describes the uncertainty in the value of $X$. If there are several events which occur with probability $p_{i}$, then the entropy of $X$ can be calculated using (1).

If there are two discrete random variables $X$ and $Y$, the joint entropy as defined in information theory is

$H_{(X, Y)}=-\sum_{x, y} p_{(x, y)} \log _{2}\left(p_{(x, y)}\right) \quad$ (bit)

where $x$ and $y$ range over all the values that $X$ and $Y$ can jointly take on. If each pair of outcomes $(x, y)$ occurs with probability $p_{(x, y)}$, the joint entropy is defined as shown in (2). If $X$ and $Y$ are independent, the joint entropy of $X$ and $Y$ is simply the sum of their individual entropies. The joint entropy is equivalent to the entropy of the joint distribution of $X$ and $Y$; in other words, it measures the entropy in a joint system of two random variables.

Definition of entropy as it is used in this paper can be applied to the physical system. Random variables can be two independent physical values. There is a special importance of distributions of physical random variables in the probability theory. Certain random variables occur often in probability theory and describe well some physical processes. In the case of the physical energy regulatory system, the random variables could represent two independent physical values, such as regulatory data and survey results, for example. It is important to note that real-world data rarely satisfy the "absolute independence" condition; however, it is useful nonetheless to model the variables as independent for the purposes of theoretical analysis.

The information entropy of a set of discrete random variables $X$ (which can take on possible values from $x_{1}$ to $x_{n}$ ) is

$H_{(X)}=E\left(I_{(X)}\right)=\sum_{i=1}^{n} p_{\left(x_{i}\right)} \log _{2}\left(\frac{1}{p_{\left(x_{i}\right)}}\right)=-\sum_{i=1}^{n} p_{\left(x_{i}\right)} \log _{2}\left(p_{\left(x_{i}\right)}\right)$ (bit)

where $I_{(X)}$ is the information content (self-information of $X$ ) that is itself a random variable, and $p\left(x_{i}\right)$ is the probability mass function of $X$, i.e.

$p\left(x_{i}\right)=\operatorname{Pr}\left(X=x_{i}\right)$

Knowing the probability of an event occurrence, information $I_{(x)}$ obtained from the event can be calculated as

$I_{(x)}=-\log _{2}\left(p_{i}\right) \quad$ (bit)

When the probabilities $p_{i}$ of all events are the same it is true that

$H_{(x)}=-\log _{2}\left(p_{i}\right) \quad$ (bit)

Information is the difference between entropy before and after an event, i.e.

$I_{(x)}=H_{(x) 1}-H_{(x) 2} \quad$ (bit)

$H_{(x)}$ is 1 when the result of an event is extremely hard to define.

A relation between entropy and negentropy should be outlined to explain in which sense the authors use term entropy in this paper. Considering theoretically, a decrease of entropy is an interesting phenomenon. The term negentropy is coined to express negative entropy. Negentropy can exist only when there is a decrease of entropy. Consequently, $N=-\Delta H$, where $N$ is negentropy and $\Delta H$ is a change of entropy (namely a difference between a posteriori value of entropy and a priori one). Therefore,

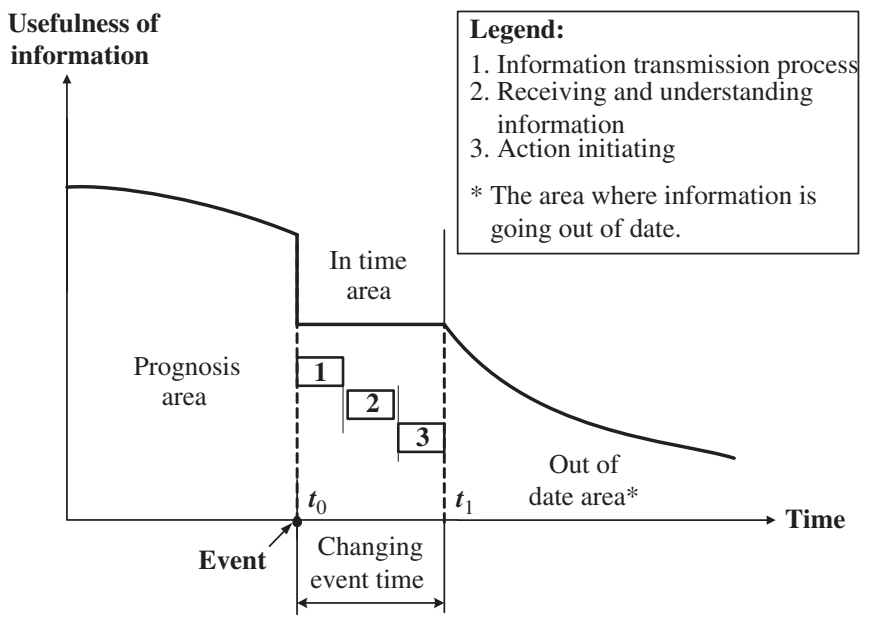

Fig. 3. Usefulness of information over time.

many papers appear the relation $N=-H$ instead the correct relation expressed above. For non-random choices or activities, the change of entropy is always negative; i.e., there is a decrease of entropy, and negentropy appears. This is equally true for human decision-making. Therefore, to use the term "production of negentropy" could be suitable.

Furthermore, the uncertainty is extremely small or zero when the total information quantity equals the entropy of the system. In this case, entropy is termed "total information quantity," and it eliminates uncertainty completely. The total information quantity is defined as

$I_{(x)}=H_{(x)}$

The total information quantity of a future event is never known before the event actually happens. Nevertheless, certain information can be collected to decrease the uncertainty associated with a given event. The metric for decreasing uncertainty depends on the nature of the system under consideration.

Usefulness of information is time-dependent (it is a typical time function as illustrated in Fig. 3) and decreases over time. This can be expressed as

$U=f(\tau)$

Furthermore, it also holds true that

$U=\frac{I_{(x)}}{H_{(x)}}$

Hence, information usefulness is proportional to negentropy that is generated by observing a certain problem. It is important that the regulatory authorities monitor events in the energy markets, collect information, analyze market trends and initiate regulatory activities in a timely manner. ${ }^{4}$ This is the main reason why regulation is not efficient if regulatory bodies are slow to act.

Analysis is essential to determine the nature of a regulatory system in the energy sector. The basic regulatory mechanism functions previously outlined include licensing, monitoring, setting and implementing tariffs and protecting consumers. The authors estimate based on their professional observations in the Croatian energy sector that the aforementioned functions encompass over $80 \%$ of all regulatory activity. Accordingly, these functions alone are sufficient to determine the entire regulatory strategy. This statement corresponds with a study that evaluated

\footnotetext{
${ }^{4}$ Initiating the regulatory activities in a timely manner is equivalent to acting within the time boundary demarcated as rectangle 3 in Fig. 3.
} 
participating $\mathrm{CIS}^{5}$ regulatory authorities [5]. The authors of this study wrote: "Thus, these regulatory authorities focus on price setting for a variety of sectors, but do not address other sectors such as licensing, dispute resolution, quality of supply, market monitoring and so forth" (p. 8).

Accordingly, the authors of this paper propose that the key functions mentioned above be analyzed with the aim of developing a regulatory framework that will minimize system entropy (uncertainty) over time.

\section{An algorithm for analyzing regulatory background context and regulatory mechanism functions}

A specific algorithm (Fig. 4) should be used for analyzing regulatory background context and regulatory mechanism functions. The proposed algorithm is highly complex and detailed, consistent with the complexity of the problem being addressed.

The algorithm consists of two main phases:

1st phase-investigating the regulatory background context, including:

- principles and methods of regulation,

- methods for defining the extent of existing regulation,

- current national regulatory body standards and organizational frameworks,

- proposal for organizational frameworks for the regulatory body,

- plan for implementation of the future regulatory body organization,

- long-term strategic goal-setting (the aims of energy market opening).

2nd phase-investigating which regulatory functions are appropriate for the given situation. A detailed examination of this phase is beyond the scope of this paper, but one possible approach is provided in Table 1.

\subsection{Algorithmic output}

As shown in Fig. 4, the algorithm generates two important outputs, namely, new information for more efficient regulatory activities and a new organizational framework for regulatory body.

\subsubsection{New information}

As expressed in (7), information is the difference between original entropy $\left(H_{(x) 1}\right)$ and entropy after the event has occurred $\left(H_{(x) 2}\right)$. In this case, the "event" refers to the complex research procedure that is implemented using the chosen algorithm.

Let $\Delta I$ denote the difference between two information quantities:

$\Delta I=I_{2}-I_{1} \quad$ (bit)

where $I_{1}$ is the quantity of information at the beginning of the research procedure (algorithmic input), and $I_{2}$ is the quantity of information after completion. Thus, $\Delta I$ represents the new quantity of information.

Furthermore, as stated in Section 3 of this paper, the usefulness of information is proportional to the negentropy that occurs following observation of a particular problem. In this case, the "problem" is how to research the necessary regulatory context for

\footnotetext{
${ }^{5}$ In the Regulatory Benchmarking Report [5], the seven CIS countries were Armenia, Georgia, Kazakhstan, the Kyrgyz Republic, Mongolia, Russia and Ukraine.
}

a certain nation's energy sector. ${ }^{6}$ Accordingly, the algorithm leads to an increase in information quantity (knowledge about the regulatory context) that is consistent with a decrease in uncertainty, equivalent to an entropy reduction (negentropy).

The quantity of information that can be collected within a given time $\tau$ is expressed as

$I_{\tau}=\frac{L}{1+a e^{-b \tau}} \quad$ (bit)

where $I_{\tau}$ is the quantity of information that is actually collected during time period $\tau, L$ is the theoretical maximum quantity of information that could be collected ( $\tau=1$, to obtain quantity $L$ ), $a$ is a coefficient, and $b$ is a second coefficient that denotes the speed of information collection.

Radosevic et al. [6] use $L=2 \times 10^{6}$ bit, $\tau=0.5, a=2$ and $b=1.385$. Given these parameters and using (12) leads to the following expression:

$I_{\tau}=\frac{2 \times 10^{6}}{1+2 e^{-1.385 .0 .5}}=\frac{2 \times 10^{6}}{2} \quad\left(\right.$ Note : $\left.e^{-1.385 .0 .5}=\frac{1}{e^{0.6925}}=0.5\right)$

The above result suggests that half of the possible maximum information is collected during the specified time period. The characteristic curves of information quantity over time for different values of $b$ are illustrated in [6].

There exists no method for measuring all the values used in (12). Parameters that cannot be measured should be assessed by experts.

The knowledge of researchers and regulatory staff increases by collecting new information during the research procedure based on the proposed algorithm. This is very important, especially in the case of small regulatory bodies with limited staff resources.

\subsubsection{Organizing a national regulatory body}

Good organizational frameworks tend to increase the efficiency of any entity. Analysts should assess efficiency by observing an organization's dynamic operation. In the case of Croatia, observation of existing regulation identified the following limitations: insufficient regulatory experience, financial issues, and a relatively short time since the regulatory body was founded (2002). There are currently 30 employees in the Croatian energy regulatory body. The authors consider that this level of staffing is inadequate for anticipated future regulatory activity loads. The Republic of Croatia as a candidate country for EU membership has to integrate relevant EU directives into its national legislation. Reform of the electricity market requires enforcement of many processes which should be carried out harmoniously [7]. Regulatory authorities in many European countries have more employees; for example, the Hungarian energy body has about a hundred staff members, while the UK has several hundred. However, the development of regulation in Croatia demands more efficient organization as well as an increase in staff numbers. The authors suggest that regulation experts use the nominated project shown in Fig. 5. This project calls for the use of network diagram techniques described in [8]. Furthermore, the regulatory body can choose and implement some specific techniques, such as decision support systems (DSS) [9], or even knowledge engineering (KE) [10], to increase operational efficiency. Two methods among network diagram techniques-the critical path method (CPM) and the project evaluation and the

\footnotetext{
${ }^{6}$ Remark: The algorithm in this paper is proposed primarily with the Croatian energy sector in mind. However, the authors consider that it may have universal applicability. In practice, it is necessary to constrain the problem by clearly stating which regulatory issues should be analyzed. The algorithm can be modified to reflect such decisions.
} 


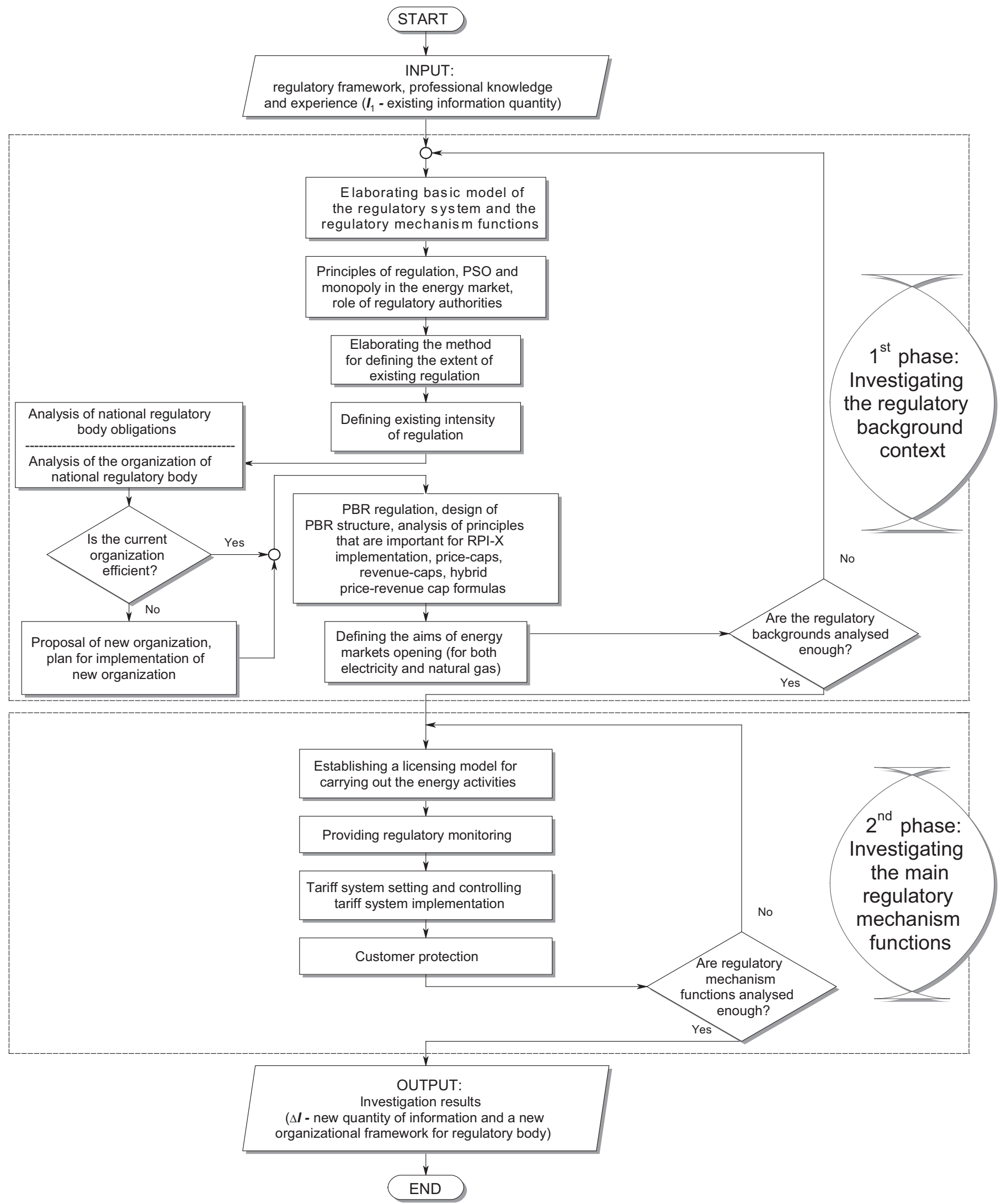

Fig. 4. Algorithm for analyzing the regulatory background context and required regulatory mechanism functions.

review technique (PERT)—-would be particularly beneficial. The authors of this paper recommend PERT because it is a method for analyzing the tasks required to complete a project and is therefore very compatible with the framework in Fig. 5. PERT focuses on the time needed to complete each task and thereby calculates the minimum time needed to complete the entire project. 
Table 1

Content of research of the regulatory mechanism functions.

Denomination of the regulatory

mechanism functions

Establishing a licensing model for

carrying out the energy activities

Providing regulatory monitoring

Tariff system setting and controlling tariff system implementation
Steps of investigating the regulatory mechanism functions

Primary and secondary goals of the licensing process

Phases in the licensing procedure

Elaborating the conditions of technical, financial and professional qualifications to be fulfilled by a legal person in order to obtain decision approving carrying out an energy activity

The Register of Licenses for carrying out energy activities issued by regulatory authorities

Rules for administrating the Summary of the Register of Licenses

Structure of the license registration number

Legislative which defines the licensing process

Disadvantages of the current licensing framework

Elaborating the criteria for upgrading the current licensing framework

Regulatory control and inspection acting

Supervising and checking the required level of technical, financial and professional qualifications based on which the license is issued for undisturbed, continuous, and safe performance of an energy activity

Regulatory approach to market monitoring issues

Setting the universal model for testing the procedure of data collecting

Development of the cost-monitoring method

Setting the logical structure of the cost-monitoring system

Characteristic of the monitoring report based on output tables of the cost-monitoring system

Regulatory authorities competences with regard to issues of setting the tariff systems for defining regulated prices for tariff customers

Regulatory authorities competences regarding monitoring the application of tariff systems

Principles for tariffing that should be promoted by the regulatory authorities

Proposal for model of tariffs setting

Description of the requirements of the participants in the energy market regarding the criterion for tariffs setting

Setting the fees for usage of transport, transmission and distribution grids

Analysis of possibilities for stranded costs compensation

Customer protection

Setting the regulatory approach in the field of customer protection

Regulatory influence on prices, investments, technical standards, quality and security of supply

Setting the customer protection model with the block-diagram of regulatory acting based on resolving customer appeals Regulatory approach to dispute resolution

Regulatory approach to the issues of risk dispersion between customers and energy undertakings

The first step in project management involves determining the required tasks and deciding on the order in which they must be completed. The project proposed in Fig. 5 is based on three main tasks: defining the claims of new organization implementation, constructing the network diagram and calculating task times, and implementing the new organizational framework. Each of those tasks comprises three steps that can be further detailed by regulatory planning experts.

\section{Extent of implemented regulation}

The authors consider that expert assessments may in many cases be more appropriate to evaluate complex systems such as those considered in this paper. This is because it may be impossible to calculate mathematically the impacts of all decisions and operations. Thus, there is no way to calculate mathematically the effects within the process of established regulatory mechanism in case of considering such complex model as the model of regulatory system. In some cases, it may be appropriate to rely on benchmarks provided by panels of experts. Such benchmarks are based on archived worldwide data and are developed using comparison/estimation approaches. Although benchmarks are commonly used today, more accurate results may be obtained in simple cases through the use of quantifiable mathematical approaches, such as those described in this paper in relation with information theory or economic issues of regulation. In more complex cases, one must rely on expert opinions, but it is imperative to use only those experts whose knowledge and experience are appropriate for the problem at hand.

In general, the maximum extent of implemented regulation can be achieved through the following:

- transparently prescribed regulatory framework;

- specific regulatory body policy and approach;

- regulatory methods and techniques that are implemented in practice.

The existing energy laws and sub-laws define the extent of allowable regulation in Croatia. It is generally appropriate for experts to define the relation between the regulatory body activities and the existing regulatory framework.

\subsection{Methods for defining the extent of implemented regulation}

Two methods should be considered:

(1) The quantity method requires mapping regulatory legislation onto specific regulatory body activities. Experts would assign an appropriate weight-index to each regulatory legal requirement. A cumulative index for each regulation act can be calculated by using these weight-indices. The regulatory authorities can use the cumulative index to prioritize all regulation acts (laws and relative sub-laws). An important prerequisite for successful implementation of the quantity 


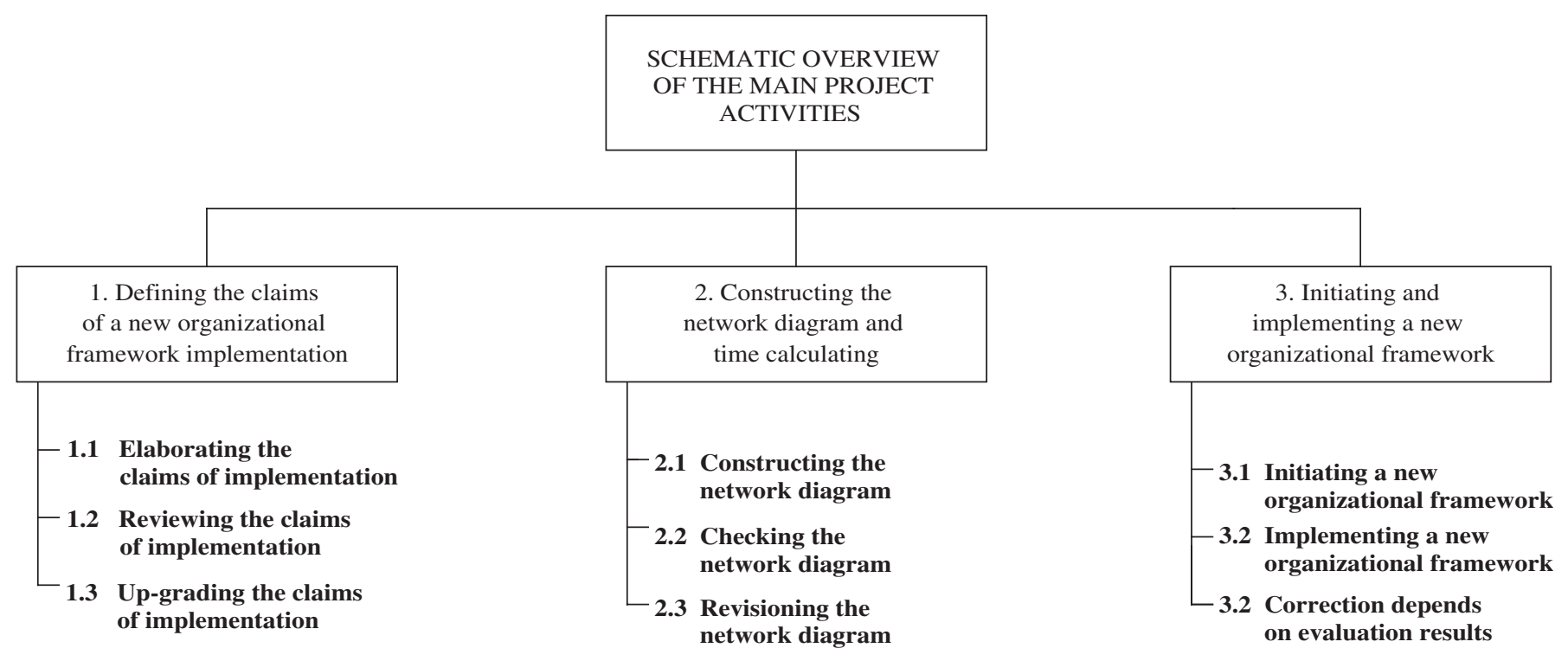

Fig. 5. Project of creating a new organizational framework.

method is long-term experience in regulatory activities and in regulatory frameworks analysis.

(2) The quality method [11,12] is based on estimates from experts. This method is not as accurate as the quantity method, but it is more practical. It can be implemented after only a few years of regulatory activity in the energy sector, and it allows for an estimation of degree of correlation between the various regulation acts.

Given the aforementioned limitations, the regulatory authorities should select a method for defining the extent of regulation on the basis of contextual evidence. Either method can be used provided that prioritization is adequately assessed and that experienced experts are employed to evaluate the legislative context. Results from a sample quality method approach are shown in Fig. 6.

There is an imbalance in the legislative framework with respect to the regulatory activities. Estimates of the extent of regulation are assessed using four levels: high, middle, low and poor [13]. In order to make these assessments, the authors of this paper analyzed the legal text within a package of energy laws [14] using the quality method. A similar approach was used to analyze agreements concerning cooperation with other governmental authorities and interactions with corporations or consumers. The result of the analysis is an overview of the Croatian energy regulatory body's competencies, duties and obligations. In general, those regulation characteristics are sufficient to determine the entire regulatory framework for the Croatian energy sector. The results are sufficient for the aforementioned extent assessments.

Fig. 6 shows that there is significant extent variation across the various laws. The law that pertains to regulation of energy activities and the regulation that specifies conditions for carrying out an energy activity [15] are the only elements that receive a "high" extent label. They are followed by the energy law, the law pertaining to the electricity market and the law pertaining to thermal energy. Extent labels of "low" and "poor" are assigned to laws that treat the gas and oil markets. The main conclusion is that different laws impose different demands on regulatory body, and these translate to different levels of regulation.

As it is mentioned before, the extent of regulation implemented in practice depends on chosen regulatory method that can be classified as follows:
Social regulation method: This approach protects society's interests. This method features standards that protect customers and employees and focuses on safeguards that protect the environment by minimizing pollution. Certain social regulation techniques impose fines on companies that do not comply with environmental or other mandates. Such fines do not influence market prices, but they may have a long-term impact on the national economy.

Regulation by negotiation: This modern regulatory method is suitable for liberalized energy markets. The regulatory authorities endeavor to act as leaders in the mediation process with the aim of solving disputes successfully. ${ }^{7}$

Performance-based regulation (PBR): PBR has existed for a hundred years, as long as the utility regulation industry itself. ${ }^{8}$ Through PBR, the regulatory authorities seek to encourage economic efficiency and endeavor to improve customer service. PBR includes different regulation techniques, such as

- Rate of return regulation (RoR), or cost plus method.

This is the basic type of economic regulation widely applied to public services, primarily in the US. This type of regulation is also known as cost of service regulation because the regulatory authorities accept only company costs that are essential for ensuring the quality of customer service. These costs are added to the basic energy price. The value of property upon which a utility is permitted to earn a specified RoR is prescribed by the regulatory authority. The base rate ${ }^{9}$ generally represents the value of the property used by the utility for providing a given service. Internal rate of return (IRR) assesses ability to report profit. IRR expresses the value ${ }^{10}$ of the discount rate at which the investment will return no economic benefit. RoR has recently been replaced with alternative approaches that

\footnotetext{
7 The intention is to achieve a "win-win" solution.

8 The sliding scale type of regulation was introduced in the US in 1906 [16]. It was first applied in the case of Boston Gas Company. This regulation is known as "the Boston sliding scale experiment", which lasted for 10 years.

${ }^{9}$ They often include working capital, cash, materials, depreciation-related deductions, accumulated deferred income taxes and investment tax credits, contributions to assist with construction, etc., depending on the method used Hence, the base rate can be calculated by any accounting method, including prudent investment, fair value, original or reproduction cost.

${ }^{10}$ The greater this value, the more competitive is the investment.
} 
R E G U L A T O R Y A C T IVITIES

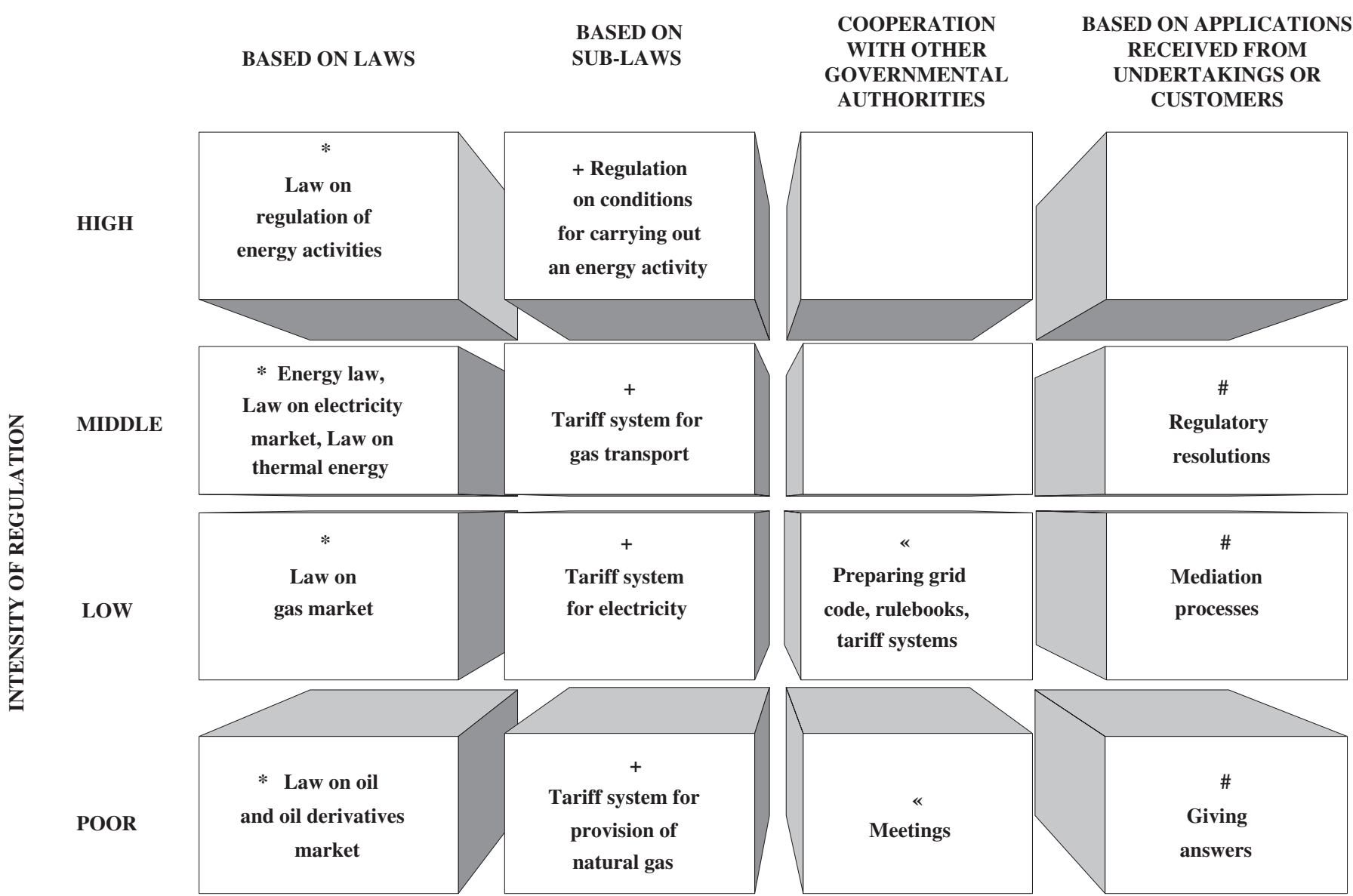

\section{LEGEND:}

* Package of six energy laws passed in 2001, 2004 and 2005

+ Secondary legislation-rulebooks, tariff systems, grid-code, etc.

« Cooperation with Ministry of Economyz, Chamber of Economy, Customer Associations, Chamber of Employers

\# Cooperation with energy undertakings and customers

Fig. 6. Extent of regulation based on the Croatian case study.

incentivize regulation (price cap and revenue cap regulation) in some US states.

- Price cap regulation and revenue cap regulation are different techniques within the economic method of regulation. ${ }^{11}$ This form of regulation is known as RPI-X regulation. RPI-X represents the inflation index minus a productivity factor. In practice, different RPI-X formulas could be implemented, but each formula should be chosen, and the final result should be approved, by the regulatory authorities. The formulas refer to:

(1) maximum price (price cap form),

(2) maximum revenue (revenue cap form),

${ }^{11}$ For example, Kaufmann and Lowry [17], Kahn [18] and Rothwell and Gomez [19] describe the economic method of regulation.
(3) both price and revenue (combined formula, i.e., hybrid revenue-price cap form).

Maximum price regulation must follow these principles:

- The regulatory authorities define the maximum price that can be charged by energy undertakings.

- The regulatory authorities define a reasonable price level transparently, before the beginning of a certain regulation period. $^{12}$

- The regulated price for a certain year is calculated by using the previous year's price with corrections via inflation index and

\footnotetext{
12 Such regulation is known as ex-ante regulation.
} 
productivity offset factors that are defined by the regulatory authorities.

Price cap formulas define the maximum price for the current year in the regulation period, which can be calculated as

$\max P_{t}=\left[\max P_{t-1}\left(1+C P I_{t}-X_{t}\right)\right]-P C F_{t}$

where $t$ is the current year in the regulation period, $t-1$ is the previous year in the regulation period, $\max P_{t}$ is the maximum price in year $t, \max P_{t-1}$ is the maximum price in year $t-1, C P I_{t}$ is the inflation index, ${ }^{13}$ i.e., annual change in prices, $X_{t}$ is the productivity offset factor in year $t$, and $P C F_{t}$ is the price corrective factor in year $t$.

On the other hand, regulatory authorities use a different form of revenue cap formulas for maximum revenue regulation. The regulatory authorities subsequently ${ }^{14}$ approve the annual revenue. Accordingly, the revenue regulation method refers to the current year. One important fact should be emphasized: revenue cap forms are often simpler for defining and controlling than are price cap forms. Furthermore, revenue cap is compatible with general accounting standards and meets regulatory needs very well. Revenue cap should generally be applied instead of price cap because price cap has a disincentivizing effect on energy efficiency investments. The main difference between revenue and price caps is that permitted revenue may change to reflect different sales levels.

An appropriate revenue cap formula that defines maximum revenue for the current year in the regulation period is

$R_{t}=\left\{\left[R_{t-1}+\left(C G A \Delta N_{\text {customers }}\right)\right]\left(1+C P I_{t}-X_{t}\right)\right\} \pm A$

where $t$ is the current year in the regulation period, $t-1$ is the previous year in the regulation period, $R_{t}$ is the authorized utility revenue in year $t, R_{t-1}$ is the authorized utility revenue in year $t-1$, $C G A$ is the customer growth adjustment factor ( $\mathrm{HRK}^{15} /$ customer), $\Delta N_{\text {customers }}$ is the annual change in the number of customers, $C P I_{t}$ is the inflation index, i.e., the annual change in prices, $X_{t}$ is the productivity offset factor in year $t$, and $A$ is an adjustment for unforeseen events (increased taxes, restructuring costs, changes in environmental laws, etc.).

As already discussed, a typical price cap formula can be transformed into a revenue cap formula. Hybrid revenue-price caps can be designed to overcome some of the problems with revenue caps, notably price volatility. Revenue caps can be set on the basis of revenues necessary to cover the costs. The basic forms of the hybrid revenue and price cap formulas are as follows:

$P_{t}=\left(1-\varepsilon_{r e l}\right) f\left(P_{t-1}\right)+\left(\varepsilon_{r e l}\right) \frac{R_{t}}{S_{t}}$

$R_{t}=\left(1-\varepsilon_{r e l}\right) P_{t} S_{t}+\left(\varepsilon_{r e l}\right) f\left(R_{t-1}\right)$

where $P_{t}$ is the price in year $t, P_{t-1}$ is the price in year $t-1, R_{t}$ is the revenue in year $t, R_{t-1}$ is the revenue in year $t-1, f\left(P_{t-1}\right)$ is the price cap component, $f\left(R_{t-1}\right)$ is the revenue cap component, $S_{t}$ represents sales in year $t$, and $\varepsilon_{r e l}$ is the relative weighting of price cap and revenue cap components.

Hence, it is possible to create multiple combined revenue-price cap formulas. The value of $\varepsilon_{r e l}$ is defined by regulatory decisionmaking and must be between $\varepsilon_{r e l}=0$ and 1 . Those limits are themselves defined by the mathematical formulas for price cap $\left(\varepsilon_{\text {rel }}=0\right)$ and for revenue cap $\left(\varepsilon_{r e l}=1\right)$.

The criterion by which the regulatory authorities determine the weighted average cost of capital (WACC) and the regulatory

\footnotetext{
${ }^{13} \mathrm{CPI}$ in the US or RPI in the UK.

14 In this respect, ex-post regulatory acting is a widely implemented approach.

15 HRK is the Croatian national currency.
}

asset base (RAB) is very important in the context of PBR. Furthermore, it is imperative for a vertically integrated utility to cover capital financing costs. There are two key components that can be utilized to assess capital-related costs:

(1) The regulatory asset base represents the regulator's assessment of the value of current investment in the regulated utility at any given time. The aim is to provide a revenue stream that has a present value equal to the regulatory asset base. Determining the initial value of the RAB is often a challenge for regulatory authorities. The opening value of the $\mathrm{RAB}^{16}$ needs to be adjusted to reflect changes for subsequent regulatory periods (this is known as a "roll-forward" process). There are two important issues in rolling forward assets: the choice of an appropriate index (using the current cost index or indexing according to general purchasing power, for example, by using RPI) and the timing of a new investment (the projected investment should be added at the beginning of a regulation period in the price cap formula and then adjusted for actual capital expenditure in the following years). Determining the RAB is important to establish the cost of capital. Treatment of depreciation should be also part of regulatory considerations.

(2) The weighted average cost of capital is the annual rate of return that investors demand for their investment. An important part of the price control review process is the estimation of WACC. The regulatory approach should require that earnings of the utility cover only the cost of capital and of running the business. It is necessary to publish transparently the contents of a fair return on capital to avoid controversy during regulatory reviews. Usually a method for determining a fair return on capital involves estimating the capital attraction rate for each component. There are many factors that regulatory authorities should consider to determinate the appropriate rate of return: the annual revaluation of assets, debt and equity ratio, returns of other utilities that have similar risks, utility status with respect to monopoly position, and specific country risk and vulnerability of the revenue stream to exchange rate movements. An appropriate formula for calculating the WACC that includes Equity plus Debt is

$W A C C=R_{e}(1-g)+g R_{d}$

where $g$ is the level of gearing, $R_{e}$ is the cost of equity finance, and $R_{d}$ is the cost of debt finance measured as risk-free rate $R_{f}$, plus a debt premium over this rate $p_{d}$.

Using the WACC, the regulatory authorities ensure that returns are equal to the opportunity cost of capital. There are several models used to estimate the cost of equity funds. ${ }^{17}$ The Capital Asset Pricing Model (CAPM) is widely used and often adopted by regulatory authorities. The following CAPM formula to measure

\footnotetext{
16 The initial value of the RAB should be the value at which the owners of the business earn a return on capital. This value has to be returned to the owners of asset over its economic-life (as depreciation). There are a range of options and numerous methods of valuing assets. The regulatory objectives for asset valuation often include: the provision of incentives for efficient investment and maintenance; the ability of the utility to finance new investment; the assurance that tariffs are no higher than is necessary; the assurance that the costs of inefficient/ imprudent investments are not borne by customers; the avoidance of rapid/large increases in tariffs; the assurance that the utility's revenue is sufficient to allow it to maintain the asset in its current condition.

17 Usual models adopted and used by regulators to estimate the cost of equity funds are: the Capital Asset Pricing Model; Price Earnings Ratio; Dividend Growth Model; Arbitrage Pricing Theory.
} 
the cost of equity is implemented in [20]:

$K_{e}=R_{f}+\beta\left(R_{m}-R_{f}\right)$

where $K_{e}$ is the cost of equity, $\beta$ is the equity beta that measures the relative risk of the utility compared to the market, $R_{f}$ is the risk-free rate on treasury securities, and $R_{m}$ is the level of market return, $\left(R_{m}-R_{f}\right)$ is the market risk premium, i.e., the amount of additional expected return that investors require to hold a broad portfolio of common stock instead of risk-free treasury securities. Finally, $\beta\left(R_{m}-R_{f}\right)$ is the market risk premium of the utility capital.

A more detailed explanation of the components of the revenue requirement is interesting but is outside the scope of this paper.

\section{Conclusions}

Taking into consideration the efficiency of regulatory mechanism is of great importance for energy activities regulation. The regulatory mechanism should be defined theoretically. Subsequently, a specific algorithm for analyzing the regulatory background context and regulatory mechanism functions should be established. The chosen algorithm should be theoretically based on information theory. Due to task complexity and limited financial and staff resources, it is desirable to analyze fewer regulatory mechanism functions.

The authors recommend setting the model of the regulatory system and comprehensive analyses of both regulatory mechanism functions and efficient organization of a regulatory body.

The four most important regulatory functions are analyzed in this paper. The authors estimate that these functions together account for more than $80 \%$ of all regulatory activity. Accordingly, the four observed functions (licensing, monitoring, tariff setting and implementation, and customer protection) define the regulatory mechanism with sufficient fidelity. An analytic approach to these issues is essential to decreasing the entropy of an implement regulatory system. Negentropy means decreased uncertainty since information quantity (knowledge important for the efficient regulation of energy activities) increases.

The Republic of Croatia is currently in negotiations for membership in the EU, and, as a consequence, it has to adopt EU directives and regulation principles. This is a strong driver for energy regulation in Croatia over the coming years. The existing organization of the national energy regulatory body should be developed to improve efficiency. There are a few different techniques that could be employed. The authors recommend the project evaluation and review technique as most suitable for the Croatian scenario. PERT analyzes the time needed to complete each task associated with the proposed project and identifies the minimum time required to complete the entire project.

Advice from regulatory experts is essential, even alongside the algorithms proposed in this paper. The extent of energy regulation can be assessed by using the quality method presented in this paper. The extent of regulation always depends on the legislative framework and on the nature of the regulatory methods and techniques used by the authorities.

\section{References}

[1] Shannon CE. A mathematical theory of communication. Bell System Technical Journal 1948;27:379-423 and 623-56.

[2] Wiener N. Cybernetics. New York: Wiley; 1948.

[3] Leff S, Rex AF. Maxwell's demon: entropy, information, computing. Princeton, NJ: Princeton University Press; 1990.

[4] Banovac E. Review of the basic elements for the Croatian Energy Regulatory Council's monitoring procedure (Monitoringgrundlagen der kroatischen Regulierungsbehörde für Energie). EW-das Magazin fur die Energie Wirtschaft 2004;1-2(103):14-6.

[5] Bjork IM, Connors CR. Regulatory benchmarking report for the CIS, produced for ERRA. Portland, ME: Pierce Atwood LLP; 2006.

[6] Radosevic D, Dusak V, Dobrenic S. Introduction in prognosis and simulation (Uvod u prognoziranje $i$ simulaciju). Varazdin: Faculty of Organization and Informatics; 1983.

[7] Banovac E, Stritof I. Analysis of applicable methods of incentive regulation in the field of distribution of electricity. WSEAS Transactions on Systems 2005;8(4):1224-32.

[8] Archibald RD, Yilloria RL. Network-based management systems: PERT/CPM. Hoboken, NJ: Wiley; 1967.

[9] Power DJ. Decision support systems: concepts and resources for managers. Westport, CT: Quorum Books; 2002.

[10] Schreiber G, Akkermans H, Anjewierden A, de Hoog R, Shadbolt N, Van de Velde W, et al. Knowledge engineering and management: the common KADS methodology. Cambridge, MA: MIT Press, Cambridge; 1999.

[11] Juran JM, Gryna MF. Quality planning and analysis. New York: McGraw-Hill; 1970.

[12] Crosby P. Cutting the cost of quality. Boston: Industrial Education Institute; 1967.

[13] Banovac E. Model of energy activities regulatory system. PhD thesis, Faculty of Electrical Engineering and Computing, University of Zagreb, Croatia, 2004.

[14] The package of energy laws: energy law, law on electricity market, law on gas market, law on oil and oil derivatives market, law on regulation of energy activities, law on thermal energy. Official Gazette of the Republic of Croatia, $68 / 01,177 / 04,42 / 05$

[15] Regulation on conditions for carrying out an energy activity. Official Gazette of the Republic of Croatia, 6/03, 94/05.

[16] Biewald B, Woolf T, Bradford P, Chernick P, Geller S, Oppenheim J. Performancebased regulation in a restructured electric industry, report prepared for the NARUC. Cambridge, MA: Synapse Energy Economics, Inc.; 1997.

[17] Kaufmann L, Lowry MN. A price cap designers handbook. Washington: Edison Electric Institute; 1995.

[18] Kahn A. The economics of regulation: principles and institutions. Cambridge, MA: MIT Press; 1988.

[19] Rothwell G, Gomez T. Electricity economics: regulation and deregulation. Piscataway: IEEE Press; 2003.

[20] Tariff system for the production, distribution and supply of thermal energy, without amounts of tariff elements. Official Gazette of the Republic of Croatia, 57/06, 88/06. 